\title{
Introduction to the Special Section of the IMEKO TC21 "Mathematical Tools in Measurements"
}

\author{
Franco Pavese
}

Torino, Italy

Section: EDITORIAL

Citation: Franco Pavese, Introduction to the Special Section of the IMEKO TC21 "Mathematical Tools in Measurements", Acta IMEKO, vol. 4, no. 4, article 1, December 2015, identifier: IMEKO-ACTA-04 (2015)-04-01

Section Editor: Franco Pavese, Torino, Italy

Received December 10, 2015; In final form December 10, 2015; Published December 2015

Copyright: (C) 2015 IMEKO. This is an open-access article distributed under the terms of the Creative Commons Attribution 3.0 License, which permits unrestricted use, distribution, and reproduction in any medium, provided the original author and source are credited

Corresponding author: Franco Pavese, email: frpavese@gmail.com

This Special Section of the IMEKO TC21 "Mathematical Tools in Measurements" is formed by a selection of extended papers that was submitted following the presentations made at the International Conference "Advanced Mathematical and Computational Tools in Metrology and Testing” (AMCTM) held in September 2014 in St. Petersburg, Russia, with an important attendance from Russia.

For this reason, this Section comprises three Russian papers out of seven. The paper of Mazin proposes the presentation of a physical quantity by a vector of the pseudo-Euclidean plane including the quantity value and its degree of uncertainty. The paper of Kurekova et al. discusses the positioning uncertainty of kinematic structures, namely of the Tricept. The paper of Kislitsyna and Malykhina deals with the modelling of an altimeter designed for soft landing on the moon, based on gamma rays scattering.

The remaining four papers well represent the worldwide attendance to the Conference. The paper of Barari and Ahmadi, from Canada, concerns coordinate metrology and introduces a novel approach to predict surface behaviour via Distribution of Geometric Deviations (DGD) to estimate the detailed deviation zone. The paper of Gonzalez, Estrada and Lira, from Mexico, discusses the modeling of a combustion chamber of a reference calorimeter used to measure the Superior Calorific Value (SCV) of natural gas. The paper of Willink, from New Zealand, discusses the important issue of the evaluation of the Type A component of uncertainty when the sample size is not predetermined, considering both the frequentist and the Bayesian methods. The paper by Pavese, from Italy, starting from a statistics of the results of Key Comparisons (KC) available on the BIPM KCDB, discusses the consequences of the results obtained, of a dominant situation where an intercomparison contains discrepant data, proposing an alternative method to the simple prior correction for the known systematic effects. 